\title{
A NEW NAME IN PSYCHOTRIA (RUBIACEAE-PSYCHOTRIEAE): PSYCHOTRIA ANDRAMONTAENSIS
}

\author{
AARON P. DAVIS \& RAFAEL GOVAERTS \\ The Herbarium, Royal Botanic Gardens, Kew, \\ Richmond, Surrey, TW9 3AE, United Kingdom
}

\section{SUMMARY}

In order to provide an accepted name for the later homonym P. bremekampiana A.P.Davis \& Govaerts we propose a new name in Psychotria: P. andramontaensis A.P.Davis \& Govaerts.

Key words: Psychotrieae, Mapouria, Psychotria, Madagascar.

\section{INTRODUCTION}

In a recent contribution (Davis et al. 2007) we transferred Indian Ocean Mapouria Aubl. to Psychotria L., making 37 new combinations and 30 new names. Unfortunately, when providing one of the new names, P. bremekampiana A.P.Davis \& Govaerts, we created a later homonym in error. We hereby correct this matter by providing a new name in Psychotria: P. andramontaensis A.P.Davis \& Govaerts.

Psychotria andramontaensis A.P. Davis \& Govaerts, nom. nov.

Mapouria discolor Bremek. (1963) 23, non Psychotria discolor (Griseb.) Rolfe (1893) 258.

Psychotria bremekampiana A.P.Davis \& Govaerts (Davis et al. 2007) 247, non Psychotria bremekampiana Steyerm. (1972) 643. - Type: Humbert, Capuron \& Cours 24786 (holo P), Madagascar (Nord-est), massif de l'Anjanaharibe, à l'ouest d'Andapa (haute Andramonta, bassin de la Lokoho), $1800 \mathrm{~m}$.

\section{REFERENCES}

Bremekamp, C.E. B. 1963. Sur quelques genres de Psychotriées (Rubiaceés) et sur leurs représentants Malgaches et Comoriens. Verh. Kon. Ned. Akad. Wetensch., Afd. Natuurk., Tweede Sect. 54, 5: 1-181.

Davis, A.P., R. Govaerts \& M. Briggs. 2007. Indian Ocean Mapouria species transferred to Psychotria (Rubiaceae-Psychotrieae). Blumea 52: 245-262.

Rolfe, R.A. 1893. Enumeration of the flowering plants and ferns of St. Vincent and adjacent islets. Bull. Misc. Inform. Kew 81: 235-296.

Steyermark, J.A. 1972. Rubiaceae. In: B. Maguire et al. (eds.), The botany of the Guayana Highland - Part IX. Mem. New York Bot. Gard. 23: 227-832. 